\title{
Deutsche Schuldenbremse: kein gutes Vorbild für Europa
}

Die politische Bedeutung der deutschen Schuldenbremse als vermeintliches Erfolgsmodell für die europäische Finanzpolitik kann kaum überschätzt werden. In der Eurokrise diente sie zunächst als Blaupause für die Verschärfung des fiskalischen Regelwerks und die angestrebte verfassungsmäßige Verankerung von Beschränkungen der öffentlichen Defizite durch den EU-Fiskalpakt. Aber auch heute noch lässt sich sagen, dass der scheinbare Erfolg der deutschen Schuldenbremse die Reform der europäischen Finanzpolitik erschwert. Dabei schien nach Beruhigung der akuten schweren Krise im Euroraum vielerorts durchaus Einigkeit zu bestehen, dass die Währungsunion vollendet werden müsse, um zukünftigen Krisen besser zu trotzen. Spätestens seit dem Fünf-Präsidenten-Report gehörte dazu auch eine deutliche Stärkung der Rolle der Finanzpolitik. Zahlreiche Beiträge aus dem akademischen Raum und schließlich die Vorschläge des französischen Präsidenten Emmanuel Macron für ein schlagkräftiges Euroraum-Budget verliehen der Diskussion danach weiteren Schwung.

In der Tat lässt sich aus ökonomischer Sicht kaum leugnen, dass die Finanzpolitik in der Währungsunion wichtiger werden muss. Aufgrund des Wegfalls der nationalen Geldpolitik kommt der nationalen Finanzpolitik eine zentrale Rolle als Stabilisator bei asymmetrischen Schocks zu, weil sich die Europäische Zentralbank (EZB) bei ihrer Zinssetzung am Euroraum-Durchschnitt orientieren muss und daher nicht auf unterschiedliche Konjunkturverläufe in einzelnen Ländern reagieren kann. Zudem muss die Fiskalpolitik die Geldpolitik bei symmetrischen Schocks besonders in schweren Krisen unterstützen, weil die Zentralbank mit ihrer herkömmlichen Zinspolitik an Grenzen stößt. Hinzu kommt, dass die jüngere Multiplikator-Debatte im Gefolge der Eurokrise gezeigt hat, dass die Finanzpolitik gerade bei schlechter Konjunktur und niedrigen Zinsen viel wirksamer ist als zuvor häufig angenommen. Dies erklärt die ungewöhnliche Tiefe der Austeritätskrise in der Währungsunion und auch, dass der Aufschwung in den südlichen Euro-Krisenländern erst seit der spürbaren Lockerung der Interpretation des fiskalischen Regelwerks durch die EU-Kommission unter JeanClaude Juncker 2015 gelang. Die Forderung nach einem Euroraum-Budget, zumindest aber nach mehr Spielräumen für die nationale Finanzpolitik ist daher sehr naheliegend; gerade erst wieder wurde sie prominent von Olivier Blanchard erhoben.

Trotz aller ökonomischen Argumente tut sich die europäische Politik aufgrund der fundamentalen Interessengegensätze und des Misstrauens zwischen den Staaten mit einer entsprechenden Reform sehr schwer. Auch wenn Deutschland mit Olaf Scholz als Bundesfinanzminister nicht mehr als Lehr- und Zuchtmeister in den Verhandlungen auftritt und die Rolle der fiskalischen Hardliner, die jedweder Lockerung der Regeln maximal kritisch gegenüberstehen, mittlerweile von den Niederlanden und der „Neuen Hanseatischen Liga“ übernommen wurde, besteht auch in weiten Teilen der deutschen Politik und Öffentlichkeit nur wenig Einsicht in die Notwendigkeit einer Reform. Zu dieser mangelnden Einsicht trägt sicher bei, dass Deutschland mit seiner Schuldenbremse sehr gut gefahren zu sein scheint: Zwar war diese immer auch ökonomisch umstritten. Kritisiert wurden die willkürliche Regeldefizitgrenze von 0,35\% des Bruttoinlandsprodukts (BIP) für den Bund und $0 \%$ für die Länder, die in der langen Frist zu unbegründbar niedrigen Schuldenstandsquoten von kaum über $10 \%$ des BIP führen würden, die unzureichende konjunkturelle Flexibilität und die fehlende Investitionsorientierung. Gerade der letzte Kritikpunkt spielte in der jüngst unter einigen deutschen Ökonomen wieder ausgebrochenen Debatte um die Schuldenbremse eine große Rolle.

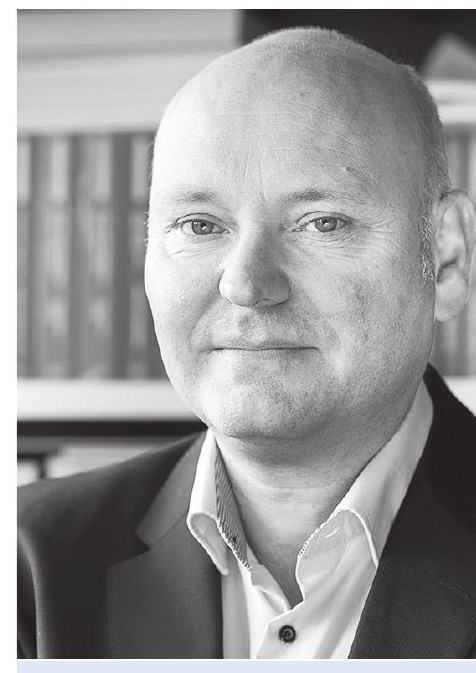

Achim Truger ist Professor für Sozioökonomie mit Schwerpunkt Staatstätigkeit und Staatsfinanzen an der Universität Duisburg-Essen, Mitglied im Sachverständigenrat zur Begutachtung der gesamtwirtschaftlichen Entwicklung und Senior Research Fellow am Institut für Makroökonomie und Konjunkturforschung (IMK) in der Hans-BöcklerStiftung. 
Dennoch sieht es so aus, als ob sich die Befürworter in ihrer ursprünglichen Haltung bestätigt sehen können, denn die öffentlichen Finanzen in Deutschland scheinen im zeitlichen und internationalen Vergleich seit Einführung der Schuldenbremse sehr gut dazustehen: Seit 2010 (mit einem gesamtstaatlichen Defizit von 4,2\% des BIP) wurde rasant konsolidiert. 2012 und 2013 war der gesamtstaatliche Haushalt in der Abgrenzung der Volkswirtschaftlichen Gesamtrechnungen (VGR) bereits annähernd ausgeglichen. Seit 2014 weist er einen Überschuss auf; 2018 lag er bei 1,7\% des BIP. Der Bundeshaushalt weist ebenfalls schon seit Jahren einen Überschuss aus und konnte die Vorgaben der Schuldenbremse sogar deutlich übererfüllen. Gleichzeitig war die deutsche Wachstums- und vor allem Beschäftigungsentwicklung im internationalen Vergleich sehr gut. Bei oberflächlicher Betrachtung mag es so aussehen, als ob die Schuldenbremse maßgeblich für die Konsolidierung verantwortlich sei und als ob die Konsolidierung der deutschen Wirtschaft nicht geschadet, möglicherweise sogar genutzt habe. Aus dieser Perspektive erschiene es folgerichtig, von den anderen Ländern der Währungsunion ebenfalls die strikte Einhaltung der Fiskalregeln zu fordern und einer Ausweitung von Spielräumen oder gar einer weiteren Risikoteilung auf europäischer Ebene sehr skeptisch gegenüberzustehen.

Bei näherer Betrachtung muss das günstige Bild der Schuldenbremse jedoch kräftig revidiert werden. Es ist unplausibel anzunehmen, die Schuldenbremse sei ursächlich für die Konsolidierungserfolge seit 2010 gewesen. Zwar wurden die Defizite gegenüber 2010 zügig reduziert. Gemessen am strukturellen, also konjunkturbereinigten und um Einmaleffekte bereinigten Budgetsaldo fiel die Konsolidierung jedoch spürbar schwächer aus. Zudem profitierten die öffentlichen Haushalte stark vom sinkenden Zinsniveau. Die scheinbar sehr eindrucksvolle strukturelle Konsolidierungsleistung des Bundes lässt sich rückblickend fast komplett auf glückliche Umstände (Konjunktur und Zinstief) sowie Sondereffekte (Rückführung von Transfers an die Sozialversicherung, Auslaufen von Konjunkturpaketen) zurückführen. Vor allem aber war die gesamtstaatliche Konsolidierung seit 2010 unter der Schuldenbremse sogar etwas schwächer ausgeprägt als in der vorangegangenen Phase von 2002 bis 2007. Die Konsolidierung seit 2010 war darüber hinaus durch ein viel günstigeres makroökonomisches Umfeld und die konjunkturschonend stärker einnahmeseitig ausgerichtete Konsolidierung begünstigt. Von 2002 bis 2007 hatten die öffentlichen Haushalte phasenweise prozyklisch gegen eine schwache Konjunktur und gegen massive Einnahmenausfälle aufgrund von Steuersenkungen ankonsolidiert - dazu war die Politik also auch ohne die damals noch gar nicht existierende Schuldenbremse fähig.

Mit der Einführung der Schuldenbremse hat die rasante Konsolidierung also wenig zu tun. Dies wird noch deutlicher, wenn man kontrafaktisch analysiert, wie sich die öffentlichen Haushalte entwickelt hätten, wenn die deutsche Konjunktur seit 2010 nicht so überaus günstig verlaufen wäre. Hätte sich die Konjunktur in Deutschland seit 2010 so schwach entwickelt wie im Euroraum insgesamt, hätte die Bundesregierung nämlich - genau wie viele andere Regierungen im Euroraum - schwer mit der Einhaltung der Grenzen des Stabilitäts- und Wachstumspakts zu kämpfen, und Bundes- und Länderhaushalte hätten wohl bis heute noch Schwierigkeiten, die Schuldenbremse einzuhalten. Ohne das 2009 und 2010 von niemandem vorhergesehene deutsche Konjunkturwunder wäre Deutschland sicher nicht zum Konsolidierungsvorbild Europas geworden und die deutsche Schuldenbremse ganz sicher nicht zur Blaupause für den europäischen Fiskalpakt. Finanzpolitisch folgt daraus vor allem eines: Die deutsche und europäische Finanzpolitik muss durch Reformen oder auch nur pragmatisches Ausnutzen bestehender Handlungsspielräume dafür Sorge tragen, dass sie sich gegebenenfalls einem möglichen kräftigen Abschwung entschlossen entgegenstemmen kann. Gelingt dies nicht, muss man sich um die Zukunft des Euro und 